\title{
PM-AM Correlation Measurements and Analysis
}

\author{
D.A. Howe, A. Hati, C. W. Nelson, and D. Lirette \\ National Institute of Standards and Technology, Time and Frequency Division \\ 325 Broadway, Boulder, CO 80305
}

\begin{abstract}
We measure and analyze effects of nonlinear mixing of phase-noise modulation (PM) and amplitude-noise modulation (AM) in an oscillating signal by real-time correlations measured in the cross power spectral density (CPSD). We outline sensitive measurements of PM-AM correlation coefficients by means of a time-averaged CPSD measurement technique. Separate but simultaneous PM and AM measurements using a two-channel cross-correlation spectrum analyzer, quantifies the relevant effects of intermodulation mixing with excellent sensitivity compared to traditional 3IM measurements and the scatter-plot correlation technique. Timeaveraged cross-spectrum measurements provide good estimates of correlation coefficients as a function of Fourier frequency (f). We use normalized PM-AM CPSD measurements of a $645 \mathrm{MHz}$ quartz-MEMs oscillator as an example and find that 1/f PMAM CPSD is exactly correlated, even for very widely differing levels of PM and AM noise, in which individual PSDs of PM and AM differ by $>40 \mathrm{~dB}$ (or, greater than a factor of 10,000). We also verify that white-PM noise has uncorrelated PM-AM CPSD.
\end{abstract}

\section{INTRODUCTION}

Energy at two frequencies propagating in an elastic medium creates intermodulation (IM) sideband frequencies. Displacements in media such as through an optical fiber, bipolar or unipolar junction, transmission line, or mechanical transducer cause distortion particularly when the medium saturates or self-levels in some way. Such distortions can generate energetic artifacts, such as phonons, that induce harmonics that alias spectral noise, introduce energy transduction at baseband, and result in a multiplicity of IM sidebands [1]. Fundamentally, two independent oscillating (sinewave) signals at frequencies $v_{1}$ and $v_{2}$ into a circuit element create harmonic signals at $\mathrm{Nv}_{1,2}, \mathrm{~N}=2,3,4, \ldots$, as well as products at $\mathrm{N} v_{1,2}-(\mathrm{N}-1) v_{2,1}$.

The following discussion is largely limited to the positivefeedback simple loop oscillator (SLO) having a narrow bandwidth frequency-determining element (resonator) compared to its on-resonance or operating frequency. The resonator of a SLO is often the largest nonlinearity affecting the PM noise, sometimes dramatically increasing it. Total harmonic distortion (THD) at $N v_{1,2}$ is principally filterable after an oscillator, whereas in-loop 3IM $\left(3^{\text {rd }}\right.$-order intermodulation) signal products, which occur for low-order $\mathrm{N}$, such as $\mathrm{N}=2$, are predominantly filterable by the resonator.

3IM universally creates undesirable PM-AM and AM-PM

Sponsored by Defense Advanced Research Projects Agency, US Government. Not subject to copyright. conversion, but this is particularly true in quartz oscillators [216]. To obtain high signal-to-noise ratio (SNR) above shot or thermal noise, most oscillators operate best just below some arbitrarily-determined onset of nonlinearity. Distortion levels of $-50 \mathrm{dBc}$ to $-70 \mathrm{dBc}$ are deemed linear enough in many cases, before the "Duffing effect", in which AM-FM conversion in the resonator rises above other 3IM effects in the feedback loop.

The principle reason to eliminate 3IM around an oscillating loop is to maintain the independence (or orthogonality) of PM and AM noises. "Orthogonality" is a critical property for reducing PM noise in the presence of AM noise, and the normalized cross power spectral density (CPSD) to be discussed is a suitable measure of orthogonality.

PM-AM correlation measurements are described in Section II. Section III contains definitions of terms. Section IV reviews three of the most common sources of signal nonlinearities that cause mixing effects between PM and AM in a SLO that cause correlation. Section V presents and interprets measurement results of a $645 \mathrm{MHz}$ miniature, lowpower quartz-MEMS oscillator.

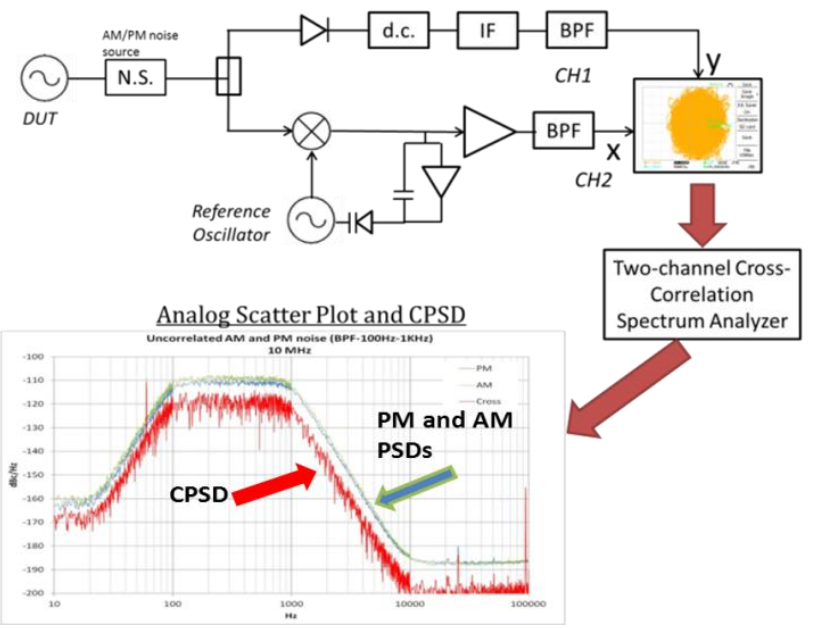

Figure 1. Two-dimensional scatter plots of analog AM and PM noise measurements $(\mathrm{CH} 1$ and $\mathrm{CH} 2$ respectively shown at top). White noise is added at the DUT-oscillator output. No PM-AM noise correlation is found, nor expected as shown by the cloudy pattern. Measuring time-averaged cross power spectral density (CPSD) is by a 2-channel FFT cross-correlation spectrum analyzer. CPSD is the grassy bottom-most trace and PM and AM are the smooth traces. The CPSD is entirely average-limited.

\section{PM-AM CORRELATION MEASUREMENT TECHNIQUES}

The noise-modulation on the phase and amplitude of an oscillating signal (carrier) are the subject here. They each create sideband power relative to the carrier power. In best-in- 
class low-noise oscillators, the noise-modulation sideband power relative to carrier offset frequency $\mathrm{f}>1 \mathrm{~Hz}$ can be -100 $\mathrm{dB}$ or lower for carrier frequencies of around $10 \mathrm{MHz}$. PM and AM noises are often assumed to be independent because deviations are (1) modeled as linear time-variant, or LTV, and (2) extremely small [17].

For assessing correlations of PM and AM noise on the carrier, that is, only noise modulations with the carrier subtracted (removed), we can measure and display the AM noise on the $\mathrm{Y}$-axis and the PM noise on the $\mathrm{X}$-axis of a 2-axis plot as shown at the top of Fig. 1. This so-called 'scatter plot' displays correlation in real time by lines and lack of correlation by the random circular cloud pattern that is shown. The scatter plot does not conveniently provide a time average, so each signal is fed to a 2-channel digital cross-correlation spectrum analyzer that computes FFTs. Implementing a cross-correlation FFT analyzer permits one to average multiple blocks of FFT spectra. Time averages can continue indefinitely until a smooth, steady-state noise level is produced.

An RF noise source (NS) with equal uncorrelated AM and PM noise is added to the oscillator, the device under test, or DUT. The signal's PM and AM noise passes through a bandpass filter (BPF) from $100 \mathrm{~Hz}<f<1 \mathrm{kHz}$ to assure uncorrelated noise of the DUT used here for example. Fig. 1

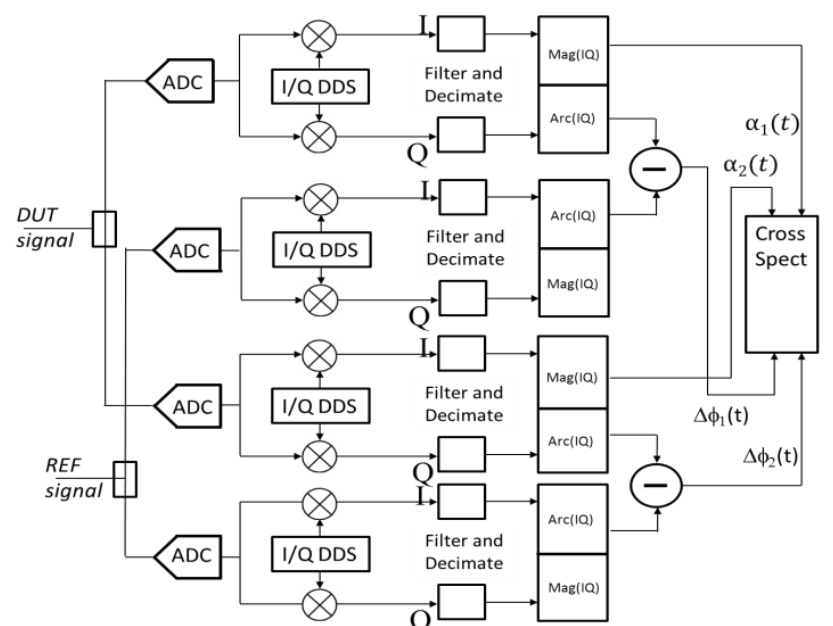

Figure 2. Digital PM and AM noise measurement systems can be constructed to simultaneously compute $S_{\phi}(f), S_{\alpha}(f)$, and $C P S D_{\phi \alpha}(f)$ [18]. A key difference between this technique and analog (Fig. 1) is that oscillator signals are digitized by a set of high-speed ADCs. PM and AM (I and Q channels) are each cross-correlated to reduce measurement-system noise before computing PM-AM CPSD, hence four separate channels are used.

shows the uncorrelated PM and AM power spectral density (PSDs are the two clean traces) and cross-correlation PSD (the grassy trace). Fig. 2 shows a digital implementation [18,19]. The degree of PM-AM correlation is characterized by the ratio of the cross-PSD $\left(C P S D_{\phi \alpha}(f)\right)$ to the geometric mean of each individual PSD $\left(\sqrt{S_{\varnothing} S_{\alpha}}\right)$. Phase and amplitude noise are exactly correlated if $C P S D_{\phi \alpha}(f)=\sqrt{S_{\varnothing} S_{\alpha}}$, thus $\log \left(\frac{C P S D_{\phi \alpha}}{\sqrt{S_{\varnothing} S_{\alpha}}}\right)$ $v s$. $\log -f$ is a convenient characterization representing the range $0-1$ of the normalized scalar correlation ratio $\frac{C P S D_{\emptyset \alpha}}{\sqrt{S_{\varnothing} S_{\alpha}}}$ that is consistent with log-log plots of PSDs.

\section{DEFINITIONS}

Henceforth, we will restrict the discussion to PM and AM noise and measurement methods that characterize PM-AM correlations by means of the CPSD. The following terms and definitions apply in this paper:

$\phi(t): \quad$ Instantaneous phase fluctuations; PM-noise function $\alpha(t)$ : Instantaneous fractional amplitude fluctuations; AM-noise function

$S_{\phi}(f)$ : One-sided double-sideband power spectral density of random phase fluctuations

$S_{\alpha}(f)$ : One-sided double-sideband power spectral density of fractional amplitude fluctuations

$C P S D_{\phi \alpha}(f)$ : One-sided double-sideband cross power spectral density of $\mathrm{S}_{\phi}(\mathrm{f})$ and $\mathrm{S}_{\alpha}(\mathrm{f})$, computed as $F F T_{\emptyset} \cdot F F T_{\alpha}^{*}$ or $F F T_{\varnothing}^{*} \cdot F F T_{\alpha}$

$\frac{C P S D_{\phi \alpha}}{\sqrt{S_{\varnothing} S_{\alpha}}}(f)$ : Normalized cross power spectral density; log plots as $10 \log C P S D_{\varnothing \alpha}-10 \log \sqrt{S_{\varnothing} S_{\alpha}}$

$\sqrt{S_{\varnothing} S_{\alpha}}(f)$ : Geometric mean of $\mathrm{S}_{\phi}(\mathrm{f})$ and $\mathrm{S}_{\alpha}(\mathrm{f})$; Average of $10 \log \mathrm{S}_{\phi}(\mathrm{f})$ and $10 \log \mathrm{S}_{\alpha}(\mathrm{f})$

\section{ORIGINS OF NONLINEAR MIXING OF PM AND AM IN AN OSCILLATING SIGNAL}

Common-mode noise sources [20,21] and spectral upconversion [22-24] will have correlated PM and AM noise caused by 3IM transduction. This section explains how 3IM causes PM and AM noises to become correlated. SLO components create two basic kinds of 3IM: (1) wideband, in which low-order harmonics $(\mathrm{N}=2,3,4)$ are not attenuated, and (2) narrowband, in which essentially all harmonics are attenuated. Wideband 3IM is caused by amplifier crossover distortion, large-signal compression or clipping, frequency comb generation through a nonlinear step-recovery diode and capacitor varactor effect, to name common causes. Narrowband 3IM self-filters its own harmonics usually by means of the high-Q, narrowband frequency determining element, i.e., the resonator. While fabrications focus on yet smaller SWaP (size,weight and power), low-power-handling, and low-phase noise, 3IM is observed inside the resonator $\mathrm{BW}$ of a simple loop oscillator,

whereas outside the resonator $\mathrm{BW}$, 3IM terms attenuate by the high-frequency rolloff of other circuit elements.

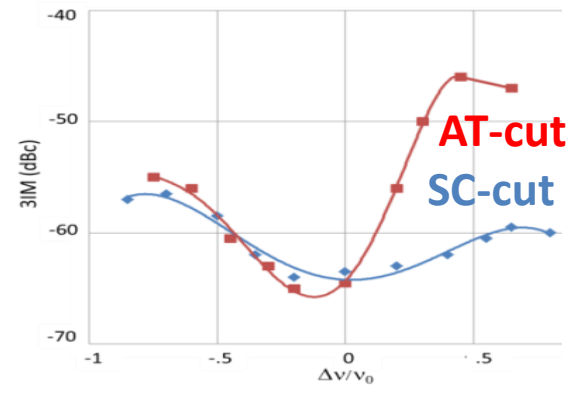

Figure 3. 3IM for $125 \mathrm{MHz} \mathrm{AT}$ - and SC-cut, fifth overtone quartz resonators [2].

\section{A. 3IM IN RESONATORS}

The connection between 3IM and PM-AM and AM-PM conversion in quartz resonators has been widely studied. Resonator 3IM has been measured to evaluate effects on various unwanted and higher-order-overtone modes to realize highest $\mathrm{Q}$ and lowest tempco in quartz resonators [2,11,25]. '3IM,' 'IM3,' and 'IIP' measurements are nomenclatures for 
measurements of intermodulation distortion of circuit elements [26]. It is either (1) the ratio of power in intermodulation sidebands to signal power for a particular input power level, or (2) power at which the 3IM distortion level equals the signal power, also called the $3^{\text {rd }}$-order intercept. Measurement (2), and the $3^{\text {rd }}$-order intercept, is a well-suited characterization for amplifiers and passive components, but not for the frequency-determining element (resonator), due to its unique Duffing effect described next. Referring to Fig. 3, we drive a resonator with two symmetric frequencies with equal powers within the resonator $\mathrm{BW}$. Intermodulation level is defined by the ratio $\mathrm{P}_{\mathrm{TT}} / \mathrm{P}_{\mathrm{IM}}$, the test tone power $\mathrm{P}_{\mathrm{TT}}\left(\right.$ at $v_{1}$ or $v_{2}$ ) to the third order intermodulation power $\mathrm{P}_{\mathrm{IM}}$ (at $2 v_{1}-v_{2}$ or $\left.2 v_{2}-v_{1}\right)$. It is often modeled as $\mathrm{P}_{\mathrm{TT}} / \mathrm{P}_{\mathrm{IM}}$ $=\mathrm{A}^{2} /\left(\delta_{\mathrm{e}}{ }^{2} \mathrm{P}_{\mathrm{TT}}{ }^{2}\right)$, where $\mathrm{A}$ is a factor depending on the resonator geometry and on the electrical circuit parameters [5]. $\delta_{\mathrm{e}}$ is an effective nonlinear constant that is calculated based on a variety of resonator parameters, and in quartz is primarily a fourth-order effect [11,27].

\section{B. Duffing Effect}

The relationship between the amplitude of a resonance and the applied force is the Duffing equation. In oscillator jargon, "Duffing" refers to the state in which a pronounced AM-toFM conversion occurs near resonator saturation (soft clipping), particularly in small resonators such as MEMs $[28,29]$. MEMs and other micro-size resonators, while very desirable for SWaP, are unfortunately prone to high nonlinear mixing due to their low saturation, or Duffing, threshold. The relationship between force and media displacement is nonlinear. The nonlinear mass-spring system can be described by $\mathrm{F}=-\mathrm{kx}\left(1+a \mathrm{x}^{2}\right)=-\mathrm{k}^{\prime} \mathrm{x}$, where $\mathrm{k}^{\prime}$ is the "effective spring constant." Since the stiffness of the nonlinear system is amplitude-dependent, it follows that the natural frequency is

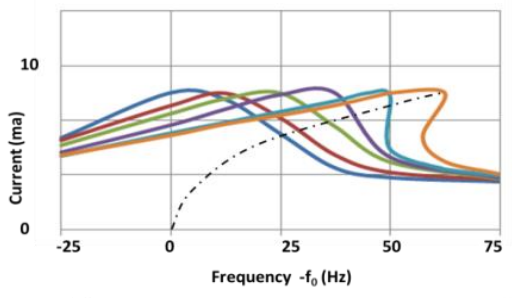
dependent on the amplitude of the vibration, i.e., the drive level. We can write the motion equation for the nonlinear system, resulting from a sinusoidal

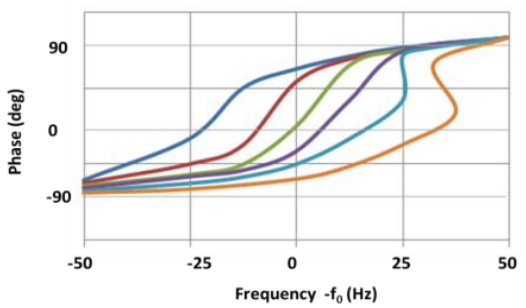

Figure 4. Normalized amplitude vs. drive current of the lumped-constant analog circuit of a $10 \mathrm{MHz}$ quartz resonator is shown at top. Sensitivity is $50 \mathrm{ppb} / \mathrm{ma}^{2}$. The phase response is shown at bottom.

driving force, as $\mathrm{mx}^{\prime \prime}+\mathrm{kx}+\mathrm{kax}^{3}=$ $\mathrm{F}_{\mathrm{d}} \cos (\omega \mathrm{t}+\phi)$. To get the electrical analog of this equation, replace the mass with an inductance, the reciprocal of the spring constant with capacitance, the displacement with the charge, and the driving force with a voltage or current. Fig. 4 shows amplitude response vs. drive current of the lumped-constant equivalent of a $10 \mathrm{MHz}$ quartz resonator. The capacitance, which is analogous to the effective spring constant, is then given by
$1 / \mathrm{C}^{\prime}=(1 / \mathrm{C})\left(1+\mathrm{aq}^{2}\right)$. The resulting equation for the sinusoidally driven LC network is $\mathrm{q}^{\prime \prime}+\omega_{\mathrm{o}}^{2} \mathrm{q}+\omega_{\mathrm{o}}^{2} \mathrm{aq}^{3}=$ $\mathrm{V} \cos (\omega \mathrm{t}+\phi)$, where $\omega_{0}=(\mathrm{LC})^{-1 / 2}$ is the natural frequency without the nonlinear term of form $q=Q \cos (\omega t)$. We arrive at an algebraic equation with $\cos ^{3}(\omega t)$ that involves terms in $\cos (3 \omega t)$, indicating that harmonics, hence intermodulation terms, are generated by the nonlinearity.

\section{3IM in Loop-oscillator Circuit Elements}

The simple loop oscillator requires signal amplification in the loop to overcome losses in the resonator and other circuit elements. All amplifiers tend to distort the input signal at power levels that optimize $\quad \mathrm{S} / \mathrm{N}$ ratio, that is, $1-2$ $\mathrm{dB}$ compression. Crossover distortion and clipping of the oscillator's loop amplifier are shown in Fig. 5. An amplifier's (bottom). For best $\mathrm{S} / \mathrm{N}$ ratio, amplifiers are often output thus operated in compression by $1-2 \mathrm{~dB}$.

contains

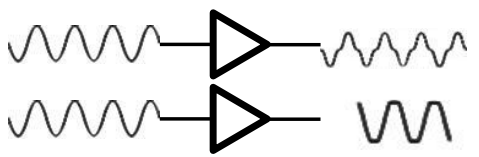

\section{Harmonic distortion causes 3IM in circuit elements}

undesirable distortion products, spurious products, and noise conversions that collectively are regarded as 3IM byproducts, which contribute to PM-AM mixing (correlation).

\section{CPSD MEASUREMENT}

A CPSD measurement of a $645 \mathrm{MHz}$ quartz-MEMs oscillator $^{1}$ is shown in Fig. 6. It is interesting to see that closeto-carrier PM-AM CPSD is exact, even for very widely differing levels of PM and AM noise. Individual PSDs of PM and AM differ by $>40 \mathrm{~dB}$, or greater than a factor of 10,000 . The resonator full $\mathrm{BW}$ is about $4 \mathrm{kHz}$ and the CPSD (shown in red) in the flicker region $f<2 \mathrm{kHz}$ means that substantially complete correlation exists inside the quartz-MEMs resonator. Correlation falls to negligible level in the white region, $f>$ $10 \mathrm{kHz}$. This result suggests that very little PM-AM mixing occurs in the loop circuit elements, with the exception of dominant mixing in the resonator. Independent admittance measurements verify that the resonator is operating in the Duffing regime [30].

\section{CONCLUSION}

We find that remedies for 3IM distortion defy simplicity in the large list of papers on quartz resonators spanning more than sixty years. Now, causes and cures of oscillating-signal PM and AM noises and strategies for reducing them become as important as strategies that are focused only on reducing PM noise. This is the first paper to quantify real-time PMAM correlations by using the CPSD. Linearity of resonators is rarely possible as elastic-resonator sizes reduce to microscopic scales. This writing supports previous assertions that, for the lowest PM noise designs, linear resonators couple with oscillating-loop elements that circumvent 3IM and its PM-AM noise correlation, which has led to significant

\footnotetext{
${ }^{1}$ Test oscillator is provided by Hughes Research Lab.
} 


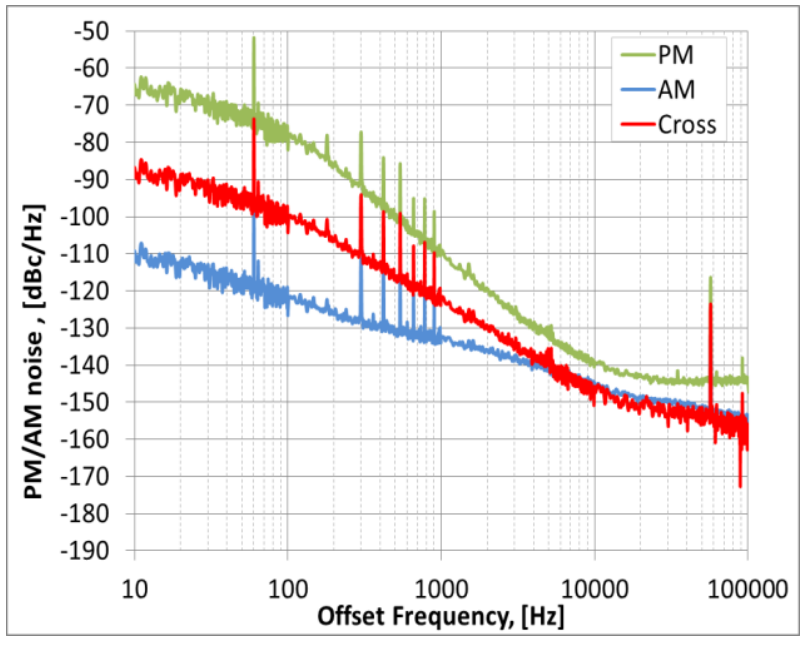

Figure 6. PM, AM, and Cross PSD of a $645 \mathrm{MHz}$ simple loop oscillator with a quartz resonator operating in an optimum quasi-linear region. Note the exact correlation between otherwise independent $\mathrm{PM}$ and $\mathrm{AM}$ deviations as seen in the cross PSD (red) in the flicker region $f<2 \mathrm{kHz}$ and no correlation in the white $f>10 \mathrm{kHz}$.

advances in ultra-low noise microwave oscillators [31-35]. Presuming that one may want to operate an oscillator at some degree of nonlinearity, knowledge of PM-AM CPSD provides a new means to quantify, isolate, and perhaps reduce or cancel noise modulation in an oscillating signal.

\section{ACKNOWLEDGMENT}

The authors gratefully acknowledge the valuable contributions and association with Harris (Chip) Moyer, Randall Kubena, Richard Joyce, Deborah Kirby, David Chang, Peter Brewer, and Robert Nagele of Hughes Research Lab. We also thank Corey Barnes, Jason DeSalvo, Neil Ashby, and Jeramy Hughes for ongoing insightful discussions. We thank Jeff Rogers of the Defense Advanced Research Project Agency (DARPA) for support of this project.

\section{REFERENCES}

[1] J. Groszkowski, "The interdependence of frequency variation and harmonic content, and the problem of constant-frequency oscillators," Proc. IRE, 21, pp. 958-981, 1933.

[2] M.D. Howard, R.C. Smythe, P.E. Morley, "Monolithic crystal filters having improved intermodulation \& power handling capability," Proc. 39th Annual Symposium on Frequency Control (FCS), pp. 491 - 503, 1985.

[3] A. Seed, "Non-linear effects in quartz resonators," 4th Intl Congress on Acoustics, Copenhagen, August, 1962.

[4] W.H. Horton \& R.C. Smythe, "Experimental investigations of intermodulation in monolithic crystal filters," Proc. 27th Annual Symposium on Frequency Control (FCS), pp. 243-245; 1973.

[5] R. L. Filler, "The amplitude-frequency effect in SC-Cut resonators," 39th ASFC, pp 311-316, 1985.

[6] R.J. Besson, J.J. Boy, M. Mourey, E.S. Ferre-Pikal, and F.L. Walls, "Phase noise limitation due to amplitude frequency effects in state-ofthe-art quartz," Proc. 50th IEEE Intl. Freq. Cont. Symp., pp. 839-843, 1996.

[7] R.C. Smythe, "Phase noise in crystal filters," Proc. 39th Annual Symposium on Frequency Control, pp. 138-139; 1985.

[8] Tiersten, H.F., "Analysis of intermodulation in rotated Y-cut quartz thickness-shear resonators," Proc. 28th FCS, pp. 1-4; 1974.

[9] Tiersten, H.F., "Analysis of intermodulation in thickness-shear and trapped-energy resonators," Acoust. Soc. Am., v. 57, no. 3 , pp. 667681; Mar. 1975.
[10] H.F. Tiersten, "An analysis of nonlinear resonance in electroded contoured AT- and SC-cut quartz crystal resonators," Proc. $38^{\text {th }}$ Ann. Symp. Freq. Cont., pp 132-140, 1984.

[11] R.C. Smythe, "Intermodulation in thickness-shear resonators," Proc. 28th AFCS, pp. 5-7; 1974.

[12] H.F. Tiersten, "An analvsis of overtone nodes in monolithic crystal filters," Proc. 30th FCS, pp. 103-108; 1976.

[13] W.H. Horton \& R.C. Smythe, "Theory of thickness-shear vibrators with extensions and applications to VHF acoustically-coupled-resonator filters," Proc. 21st FCS, pp. 160-178; 1967.

[14] R.C.Smythe, "VHF Monolithic Filters," Rpt. No. ECOM-72-0025-F, U.S. Army Electronics Command, Ft. Monmouth, NJ, Feb., 1976.

[15] R.C. Smythe, "Bulk acoustic wave filters," in Precision Frequency Control (E.A. Gerber and A.D. Ballato, ed's), pp. 188-228; Academic Press, New York, 1985.

[16] R.C. Smythe and H.F. Tiersten, "An approximate expression for the motional capacitance of a lateral field resonator," Trans. IEEE on Ultrasonics, Ferroelectrics, and Frequency Control, v. UFFC-35, No. 3, pp. 435-436, May, 1988.

[17] A. Hajimiri, T. H. Lee. "A general theory of phase noise in electrical oscillators." IEEE Journal of Solid-State Circuits, 33, no. 2, pp179194, 1998.

[18] C. W. Nelson and D. A. Howe, "A sub-sampling digital AM/PM noise measurement system," Proc. 2012 IEEE International Frequency Control Symposium (these proceedings).

[19] L. B. Ruppalt, D. R. McKinstry, K. C. Lauritzen, A. K. Wu, S. A. Phillips, and S. H. Talisa, "Simultaneous digital measurement of phase and amplitude noise," in Frequency Control Symposium (FCS), 2010 IEEE International, 2010, pp. $97-102$.

[20] E.S. Ferre-Pikal, M.C. Aramburo, F.L. Walls, and K.M. Lakin, "1/f frequency noise of 2-GHz high-Q thin-film sapphire resonators," IEEE T. Ultrason. Ferr., pp. 506-510, 2001.

[21] F.L. Walls, E.S. Ferre-Pikal, and S.R. Jefferts, "1/f PM and AM noise in amplifiers and oscillators," Proc. 2001 ICTF Conf., pp. 226-240.

[22] E. Hegazi and A. A. Abidi, "Varactor characteristics, oscillator tuning curves, and AM-FM conversion," IEEE Journal of Solid-State Circuits, 2003.

[23] V. N. Kuleshov and T. I. Boldyreva, "1/f AM and PM noise in bipolar transistor amplifiers: sources, ways of influence, techniques of reduction," Proc. 1997 IEEE International Frequency Control Symposium pp. 446-455.

[24] V. N. Kuleshov, "PM and AM noise in nonlinear BJT amplifiers," Proc. 13 ${ }^{\text {th }}$ Europiean Frequency and Time Forum and 1999 IEEE International Frequency Control Symposium, pp. 1180-1183.

[25] M. Driscoll, "Oscillator AM-to-FM noise conversion due to the dynamic frequency-drive sensitivity of the crystal resonator," Proc. 2008 IEEE Intl. Freq. Cont. Symp., pp. 672 - 676, 2008.

[26] K. A. Remley, D. F. Williams, M. Dominique, M. P. Schreurs, J. Wood. "Simplifying and interpreting two-tone measurements." IEEE Transactions on Microwave Theory and Techniques, 52. no. 11, pp2576-2584, 2004.

[27] J. J. Gagnepain, "Nonlinear properties of quartz crystal and quartz resonators: a review," Proc. 35th FCS, pp. 14-30, 1981.

[28] L. He, Y. P. Xu, M. Palaniapan, "A state-space phase noise model for nonlinear MEMS oscillators employing automatic amplitude control," IEEE TRANS CIRCUITS AND SYSTEMS, 57, no. 1, 2010.

[29] S. Zaitsev, R. Almog, O. Shtempluck, E. Buks, "Nonlinear dynamics in nanomechanical oscillators," Proceedings of the International Conference on MEMS, NANO, and Smart Systems, IEEE Computer Society, 2005.

[30] H. Moyer, R. Nagele, R. Kubena, R. Joyce, D. Kirby, P. Brewer, D. Chang, "Nonlinear behavior of an UHF quartz resonator in an oscillating system," Proc. 2012 IEEE International Frequency Control Symposium (these proceedings).

[31] A. Sen Gupta, D.A. Howe, C. Nelson, A. Hati, F.L. Walls, and J.F. Garcia Nava, "High spectral purity microwave oscillator: Design using conventional air-dielectric cavity," IEEE T. Ultrason. Ferr. (UFFC), 51, pp. 1225-1231, 2004. 
[32] United States Patent No.
http://tf.boulder.nist.gov/general/pdf $/ 2180 . p d f$

[33] E.N Ivano and M.E Tobar, "Low phase-noise sapphire crystal microwave oscillators: Current status," IEEE UFFC 56, 263 (2009).

[34] S. Grop, P.Y. Bourgeois, R. Boudot, Y. Kersale, E. Rubiola and V. Giordano, "10 GHz cryocooled sapphire oscillator with extremely low phase noise", IEEE Electronics Letters 46, pp. 420 (2010).
[35] D. P. Tsarapkin, "Phase noise in microwave bridge oscillators," Proc 2005 IEEE International Frequency Control Symposium and PTTI Meeting 29-31 Aug., pp. 534-538. 